\title{
Absolutely continuous operators on function spaces and vector measures
}

\author{
Marian Nowak
}

Received: 15 May 2012 / Accepted: 29 May 2012 / Published online: 26 June 2012

(C) The Author(s) 2012. This article is published with open access at Springerlink.com

\begin{abstract}
Let $(\Omega, \Sigma, \mu)$ be a finite atomless measure space, and let $E$ be an ideal of $L^{0}(\mu)$ such that $L^{\infty}(\mu) \subset E \subset L^{1}(\mu)$. We study absolutely continuous linear operators from $E$ to a locally convex Hausdorff space $(X, \xi)$. Moreover, we examine the relationships between $\mu$-absolutely continuous vector measures $m: \Sigma \rightarrow X$ and the corresponding integration operators $T_{m}: L^{\infty}(\mu) \rightarrow X$. In particular, we characterize relatively compact sets $\mathcal{M}$ in $c a_{\mu}(\Sigma, X)$ (= the space of all $\mu$-absolutely continuous measures $m: \Sigma \rightarrow X$ ) for the topology $\mathcal{T}_{s}$ of simple convergence in terms of the topological properties of the corresponding set $\left\{T_{m}: m \in \mathcal{M}\right\}$ of absolutely continuous operators. We derive a generalized Vitali-Hahn-Saks type theorem for absolutely continuous operators $T: L^{\infty}(\mu) \rightarrow X$.
\end{abstract}

Keywords Function spaces - Absolutely continuous operators · Integration operators · Countably additive vector measures $\cdot$ Absolutely continuous vector measures · Mackey topologies · Order-bounded topology

Mathematics Subject Classification (2010) $\quad 46 \mathrm{G} 10 \cdot 28 \mathrm{~A} 33 \cdot 28 \mathrm{~A} 25 \cdot 47 \mathrm{~B} 38$

\section{Introduction and terminology}

For terminology concerning vector lattices and function spaces we refer the reader to [1], [2], [10]. Throughout the paper we assume that $(\Omega, \Sigma, \mu)$ is a complete finite atomless measure space and $L^{0}(\mu)$ denotes the corresponding space of $\mu$-equivalence classes of all $\Sigma$-measurable real valued-functions defined on $\Omega$. Let $E$ be an

M. Nowak $(\varangle)$

Faculty of Mathematics, Computer Science and Econometrics, University of Zielona Góra, ul. Szafrana 4A, 65-516 Zielona Gora, Poland e-mail: M.Nowak@wmie.uz.zgora.pl 
ideal of $L^{0}(\mu)$ such that $L^{\infty}(\mu) \subset E \subset L^{1}(\mu)$, and let $E^{\sim}$ and $E_{n}^{\sim}$ stand for the order dual and order continuous dual of $E$ respectively. Then $E_{n}^{\sim}$ separates the points of $E$ and it can be identified with the Köthe dual $E^{\prime}$ of $E$ through the mapping $E^{\prime} \ni v \mapsto \varphi_{v} \in E_{n}^{\sim}$, where $\varphi_{v}(u)=\int_{\Omega} u v d \mu$ for all $u \in E$. It is known that the Mackey topology $\tau\left(E, E_{n}^{\sim}\right)\left(=\tau\left(E, E^{\prime}\right)\right)$ is a locally solid Lebesgue topology.

The so-called order-bounded topology $\tau_{0}$ can be defined on $E$ as the finest locally convex topology on $E$ for which every order interval in $E$ is a bounded set (see [11]). A local base $\mathcal{B}_{0}$ at zero for $\tau_{0}$ is the class of all absolutely convex subsets of $E$ that absorb all order bounded sets in $E$. Then $\tau_{0}$ coincides with the Mackey topology $\tau\left(E, E^{\sim}\right)$. Note that if $u_{n}, u \in E$ and $u_{n} \rightarrow u$ uniformly on $\Omega$, then $u_{n} \rightarrow u$ for $\tau_{0}$.

From now on we assume that $(X, \xi)$ is a locally convex Hausdorff space (for short, lcHs) and let $\mathcal{P}_{\xi}$ denote the set of all $\xi$-continuous seminorms on $X$. By $X_{\xi}^{\prime}$ we denote the topological dual of $(X, \xi)$. We denote by $\sigma(L, K)$ and $\tau(L, K)$ the weak topology and the Mackey topology on $L$ with respect to a dual pair $\langle L, K\rangle$.

Recall that a linear operator $T: E \rightarrow X$ is said to be order-bounded (resp. orderweakly compact), if for each $u \in E^{+}$, the set $T([-u, u])$ is $\xi$-bounded (resp. relatively $\sigma\left(X, X_{\xi}^{\prime}\right)$-compact) in $X$ (see [6]).

Proposition 1.1 For a linear operator $T: E \rightarrow X$ the following statements are equivalent:

(i) $T$ is order-bounded.

(ii) $T$ is $\left(\tau_{0}, \xi\right)$-continuous.

Proof (i) $\Longrightarrow$ (ii) Assume that $T$ is order-bounded. Let $p \in \mathcal{P}_{\xi}$ and $\varepsilon>0$. We shall show that there is $V \in \mathcal{B}_{0}$ such that $T(V) \subset B_{p}(\varepsilon)(=\{x \in X: p(x) \leq \varepsilon\})$. Indeed, let $V=T^{-1}\left(B_{p}(\varepsilon)\right)$. Since $T(V) \subset T\left(T^{-1}\left(B_{p}(\varepsilon)\right) \subset B_{p}(\varepsilon)\right.$, it is enough to show that $V$ absorbs every order interval in $E$. Given $u \in E^{+}$there is $r_{u}>0$ such that $T([-u, u]) \subset B_{p}\left(r_{u}\right)$. Then for $\lambda_{u}=\frac{\varepsilon}{r_{u}}$ and for all $v \in[-u, u]$ we get $p\left(T\left(\lambda_{u} v\right)\right)=\lambda_{u} p(T(v)) \leq \varepsilon$, so $\lambda_{u} v \in V$. This means that $\lambda_{u}[-u, u] \subset V$, i.e., $V$ absorbs $[-u, u]$, as desired.

(ii) $\Longrightarrow$ (i) Assume that $T$ is $\left(\tau_{0}, \xi\right)$-continuous and $p \in \mathcal{P}_{\xi}$. Then there is $V_{p} \in \mathcal{B}_{0}$ such that $T\left(V_{p}\right) \subset B_{p}(1)$. Given $u \in E^{+}$there exists $\lambda_{u}>0$ such that $\lambda_{u}[-u, u] \subset$ $V_{p}$. Hence $T\left(\lambda_{u}[-u, u]\right) \subset T\left(V_{p}\right) \subset B_{p}(1)$, so $T([-u, u]) \subset B_{p}\left(\frac{1}{\lambda_{u}}\right)$. It follows that the set $T([-u, u])$ is $\xi$-bounded in $X$.

Following [13] a linear operator $T: E \rightarrow X$ is said to be absolutely continuous if for each $u \in E, T\left(\mathbb{1}_{A_{n}} u\right) \rightarrow 0$ for $\xi$ whenever $\mu\left(A_{n}\right) \rightarrow 0,\left(A_{n}\right) \subset \Sigma$. Absolutely continuous operators on Orlicz spaces and Frechét function spaces have been examined by Orlicz and Wnuk (see [12,13]).

In Sect. 2 we study absolutely continuous operators $T: E \rightarrow X$. We show that a linear operator $T: E \rightarrow X$ is absolutely continuous if and only if $T$ is $\left(\tau\left(E, E_{n}^{\sim}\right), \xi\right)$ continuous. We characterize relatively compact sets in the space $\mathcal{L}_{\tau, \xi}(E, X)$ of all $\left(\tau\left(E, E_{n}^{\sim}\right), \xi\right)$-continuous linear operators $T: E \rightarrow X$, provided with the topology of simple convergence. In Sect. 3 we examine the relationships between $\mu$-absolutely continuous vector measures $m: \Sigma \rightarrow X$ and the corresponding integration operators $T_{m}: L^{\infty}(\mu) \rightarrow X$. 


\section{Absolutely continuous operators on function spaces}

We start with the following result.

Proposition 2.1 Assume that $T: E \rightarrow X$ is an absolutely continuous linear operator. Then $T$ is $\left(\tau_{0}, \xi\right)$-continuous.

Proof In view of Proposition 1.1 it is sufficient to show that $T([-u, u])$ is $\xi$-bounded in $X$ for every $u \in E^{+}$. For this purpose one can repeat the proof of Theorem 1 in [13].

Now we present a characterization of absolutely continuous operators on $E$.

Proposition 2.2 For a linear operator $T: E \rightarrow X$ the following statements are equivalent:

(i) $x^{\prime} \circ T \in E_{n}^{\sim}$ for each $x^{\prime} \in X_{\xi}^{\prime}$.

(ii) $T$ is $\left(\sigma\left(E, E_{n}^{\sim}\right), \sigma\left(X, X_{\xi}^{\prime}\right)\right)$-continuous.

(iii) $T$ is $\left(\tau\left(E, E_{n}^{\sim}\right), \xi\right)$-continuous.

(iv) $T$ is smooth, i.e., $T\left(u_{\alpha}\right) \rightarrow 0$ for $\xi$ whenever $u_{\alpha} \stackrel{\text { (o) }}{\longrightarrow} 0$ in $E$.

(v) $T$ is $\sigma$-smooth, i.e., $T\left(u_{n}\right) \rightarrow 0$ for $\xi$ whenever $u_{n} \stackrel{(\mathrm{o})}{\longrightarrow} 0$ in $E$.

(vi) $T$ is absolutely continuous.

Proof (i) $\Longleftrightarrow$ (ii) See [1, Theorem 9.26].

(ii) $\Longrightarrow$ (iii) Assume that $T$ is $\left(\sigma\left(E, E_{n}^{\sim}\right), \sigma\left(X, X_{\xi}^{\prime}\right)\right)$-continuous. It follows that $T$ is $\left(\tau\left(E, E_{n}^{\sim}\right), \tau\left(X, X_{\xi}^{\prime}\right)\right)$-continuous (see [1, Exercise 11, p. 149]), and hence $T$ is $\left(\tau\left(E, E_{n}^{\sim}\right), \xi\right)$-continuous because $\xi \subset \tau\left(X, X_{\xi}^{\prime}\right)$.

(iii) $\Longrightarrow$ (iv) Assume that $T$ is $\left(\tau\left(E, E_{n}^{\sim}\right), \xi\right)$-continuous, and let $\left(u_{\alpha}\right)$ be a net in $E$ such that $u_{\alpha} \stackrel{(\mathrm{o})}{\longrightarrow} 0$ in $E$. Then $u_{\alpha} \rightarrow 0$ for $\tau\left(E, E_{n}^{\sim}\right)$ because $\tau\left(E, E_{n}^{\sim}\right)$ is a Lebesgue topology on $E$. Hence $T\left(u_{\alpha}\right) \rightarrow 0$ for $\xi$, as desired.

(iv) $\Longrightarrow$ (v) It is obvious.

$(\mathrm{v}) \Longleftrightarrow$ (vi) It is enough to repeat the reasoning in the proof of Proposition 4 in [13] and use Proposition 2.1 and the fact that $u_{n} \rightarrow 0$ in $E$ for $\tau_{0}$ whenever $u_{n} \rightarrow 0$ uniformly on $\Omega$.

(v) $\Longrightarrow$ (i) It is obvious.

Corollary 2.3 Every absolutely continuous operator $T: E \rightarrow X$ is order-weakly compact.

Proof Note that for each $u \in E^{+}$, the order interval [-u,u] in $E$ is relatively $\sigma\left(E, E_{n}^{\sim}\right)$-compact because $\tau\left(E, E_{n}^{\sim}\right)$ is a Lebesgue topology (see [2], Theorem 6.62]). Hence by Proposition 2.2 the set $T([-u, u])$ is relatively $\sigma\left(X, X_{\xi}^{\prime}\right)$-compact in $X$, as desired.

Let $\mathcal{L}_{\tau, \xi}(E, X)$ stand for the space of all $\left(\tau\left(E, E_{n}^{\sim}\right), \xi\right)$-continuous linear operators from $E$ to $X$, equipped with the topology $\mathcal{T}_{s}$ of simple convergence. Let $\mathcal{P}_{\xi}$ be the family of all $\xi$-continuous seminorms on $X$. Then $\mathcal{T}_{s}$ is generated by the family $\left\{q_{p, u}\right.$ : $\left.p \in \mathcal{P}_{\xi}, u \in E\right\}$ of seminorms, where $q_{p, u}(T)=p(T(u))$ for all $T \in \mathcal{L}_{\tau, \xi}(E, X)$.

The following result will be of importance (see [15, Theorem 2]). 
Theorem 2.4 Let $\mathcal{K}$ be a $\mathcal{T}_{s}$-compact subset of $\mathcal{L}_{\tau, \xi}$. If $C$ is a $\sigma\left(X_{\xi}^{\prime}, X\right)$-closed and $\xi$-equicontinuous subset of $X_{\xi}^{\prime}$, then $\left\{x^{\prime} \circ T: T \in \mathcal{K}, x^{\prime} \in C\right\}$ is a $\sigma\left(E_{n}^{\sim}, E\right)$-compact subset of $E_{n}^{\sim}$.

Now we can state a characterization of relative $\mathcal{T}_{s}$-compactness in $\mathcal{L}_{\tau, \xi}(E, X)$.

Theorem 2.5 Let $\mathcal{K}$ be a subset of $\mathcal{L}_{\tau, \xi}(E, X)$. Then the following statements are equivalent:

(i) $\mathcal{K}$ is relatively $\mathcal{T}_{s}$-compact.

(ii) $\mathcal{K}$ is $\left(\tau\left(E_{n}^{\sim}, E\right), \xi\right)$-equicontinuous and for each $u \in E$, the $\operatorname{set}\{T(u): T \in \mathcal{K}\}$ is relatively $\xi$-compact in $X$.

Proof (i) $\Longrightarrow$ (ii) Assume that $\mathcal{K}$ is relatively $\mathcal{T}_{s}$-compact. Let $W$ be an absolutely convex and $\xi$-closed neighbourhood of 0 for $\xi$ in $X$. Then the polar $W^{0}$ of $W$ (with respect to the dual pair $\left\langle E, E_{\xi}^{\prime}\right\rangle$ ), is a $\sigma\left(X_{\xi}^{\prime}, X\right)$-closed and $\xi$-equicontinuous subset of $X_{\xi}^{\prime}$ (see [1, Theorem 9.21]). Then by Theorem 2.4 the set $H=\left\{x^{\prime} \circ T: T \in \mathcal{K}, x^{\prime} \in W^{0}\right\}$ in $E_{n}^{\sim}$ is $\sigma\left(E_{n}^{\sim}, E\right)$-compact. Hence in view of the Nakamo theorem (see [2, Corollary 6.31]) the $\sigma\left(E_{n}^{\sim}, E\right)$-closed absolutely convex hull (abs conv $\left.\mathrm{H}\right)^{-}$of $H$ is $\sigma\left(E_{n}^{\sim}, E\right)$ compact in $E_{n}^{\sim}$. The the polar $V=\left((\operatorname{absconv} H)^{-}\right)^{0}$ (with respect to the dual pair $\left.\left\langle E, E_{n}^{\sim}\right\rangle\right)$ is a $\tau\left(E, E_{n}^{\sim}\right)$-neighbourhood of 0 in $E$ and $H \subset V^{0}$. Then for each $T \in \mathcal{K}$ we have that $\left\{x^{\prime} \circ T: x^{\prime} \in W^{0}\right\} \subset V^{0}$, i.e., if $x^{\prime} \in W^{0}$, then $\left|x^{\prime}(T(u))\right| \leq 1$ for all $u \in V$. This means that for each $T \in \mathcal{K}$ we have $W^{0} \subset T(V)^{0}$. Hence $T(V) \subset$ $T(V)^{00} \subset W^{00}=W$ for each $T \in \mathcal{K}$, i.e., $\mathcal{K}$ is $\left(\tau\left(E, E_{n}^{\sim}\right), \xi\right)$-equicontinuous.

Clearly, for each $u \in E$, the set $\{T(u): T \in \mathcal{K}\}$ is relatively $\xi$-compact in $X$.

(ii) $\Longrightarrow$ (i) It follows from [3, Chap. 3, § 3.4, Corollary 1], [4, Chap. 3.2.2, Corollary, p. 89].

Corollary 2.6 Assume that $\mathcal{K}$ is a relatively $\mathcal{T}_{s}$-compact subset of $\mathcal{L}_{\tau, \xi}(E, X)$. Then $\mathcal{K}$ is uniformly $\mu$-absolutely continuous, i.e., for each $u \in E$ and $p \in \mathcal{P}_{\xi}$ we have

$$
\sup _{T \in \mathcal{K}} p\left(T\left(\mathbb{1}_{A_{n}} u\right)\right) \longrightarrow 0 \text { whenever } \mu\left(A_{n}\right) \longrightarrow 0,\left(A_{n}\right) \subset \Sigma \text {. }
$$

Proof In view of Theorem $2.4, \mathcal{K}$ is $\left(\tau\left(E, E_{n}^{\sim}\right), \xi\right)$-equicontinuous. Let $p \in \mathcal{P}_{\xi}$ and $\varepsilon>0$ be given. Then there exists a $\tau\left(E, E_{n}^{\sim}\right)$-neigbourhood $V$ of 0 in $E$ such that for each $T \in \mathcal{K}$ we have $p(T(u)) \leq \varepsilon$ for all $u \in V$. Let $u \in E$ and $\mu\left(A_{n}\right) \rightarrow 0$ and let $u_{n}=\mathbb{1}_{A_{n}} u$ for $n \in \mathbb{N}$. Note that $u_{n} \rightarrow 0(\mu)$ and $\left|u_{n}(\omega)\right| \leq|u(\omega)| \mu$-a.e. for all $n \in \mathbb{N}$. Hence by the Riesz theorem for every subsequence $\left(u_{k_{n}}\right)$ of $\left(u_{n}\right)$ there exists a subsequence $\left(u_{l_{k_{n}}}\right)$ of $\left(u_{k_{n}}\right)$ such that $u_{l_{k_{n}}}(\omega) \rightarrow 0 \mu$-a.e. This means that $u_{l_{k_{n}}} \stackrel{(\mathrm{o})}{\longrightarrow} 0$ in $E$ (see [10, Chap. 10, §1]). Hence $u_{l_{k_{n}}} \rightarrow 0$ for $\tau\left(E, E_{n}^{\sim}\right)$ because $\tau\left(E, E_{n}^{\sim}\right)$ is a Lebesgue topology. It follows that $u_{n} \rightarrow 0$ for $\tau\left(E, E_{n}^{\sim}\right)$. Then there exists $n_{\varepsilon} \in \mathbb{N}$ such $u_{n} \in V$ for $n \geq n_{\varepsilon}$, and hence $\sup _{T \in \mathcal{K}} p\left(T\left(\mathbb{1}_{A_{n}} u\right)\right) \leq \varepsilon$ for $n \geq n_{\varepsilon}$.

\section{Absolutely continuous vector measures}

Let $(X, \xi)$ be a quasicomplete lcHs and $m: \Sigma \rightarrow X$ be a $\xi$-bounded vector measure (i.e., the range of $m$ is $\xi$-bounded in $X$ ) and $m(A)=0$ if $\mu(A)=0, A \in \Sigma$ (in symbols, $m \ll \mu)$. 
For $u \in L^{\infty}(\mu)$ let $\|u\|_{\infty}=\operatorname{ess} \sup _{\omega \in \Omega}|u(\omega)|$. Given $u \in L^{\infty}(\mu)$, let $\left(s_{n}\right)$ be a sequence in $\mathcal{S}(\mu)$ (= the space of all $\mu$-simple functions on $\Omega$ ) such that $\left\|u-s_{n}\right\|_{\infty} \rightarrow 0$ (see [10, Chap. 1, §6, Theorem 3]). Define

$$
\int_{\Omega} u d m:=\xi-\lim \int_{\Omega} s_{n} d m .
$$

Then the integral $\int_{\Omega} u d m$ is well defined and the corresponding integration operator $T_{m}: L^{\infty}(\mu) \rightarrow X$ given by $T_{m}(u)=\int_{\Omega} u d m$ is $\left(\|\cdot\|_{\infty}, \xi\right)$-continuous and linear, and for each $x^{\prime} \in X_{\xi}^{\prime}$,

$$
x^{\prime}\left(\int_{\Omega} u d m\right)=\int_{\Omega} u d\left(x^{\prime} \circ m\right) \text { for } u \in L^{\infty}(\mu),
$$

(see [9], [14, Lemma 6]). Conversely, let $T: L^{\infty}(\mu) \rightarrow X$ be a $\left(\|\cdot\|_{\infty}, \xi\right)$-continuous linear operator, and let $m(A)=T\left(\mathbb{1}_{A}\right)$ for $A \in \Sigma$. Then $m: \Sigma \rightarrow X$ is a $\xi$-bounded vector measure such that $m \ll \mu$ (called the representing measure of $T$ ) and $T_{m}(u)=T(u)$ for all $u \in L^{\infty}(\mu)$.

An important example of a quasicomplete lcHs is the space $\mathcal{L}(Y, Z)$ of all bounded linear operators between Banach spaces $Y$ and $Z$, provided with the strong operator topology.

Recall that a vector measure $m: \Sigma \rightarrow X$ is said to be $\mu$-absolutely continuous $m\left(A_{n}\right) \rightarrow 0$ for $\xi$ whenever $\mu\left(A_{n}\right) \rightarrow 0,\left(A_{n}\right) \subset \Sigma$ (see [5, Definition 3, p. 11]).

Now we characterize $\mu$-absolutely continuous measures in terms of the properties of the corresponding integration operators.

Proposition 3.1 Assume that $(X, \xi)$ is a quasicomplete lcHs. Let $m: \Sigma \rightarrow X$ be $a \xi$-bounded vector measure such that $m \ll \mu$. Then the following statements are equivalent:

(i) $x^{\prime} \circ m \in c a_{\mu}(\Sigma)$ for each $x^{\prime} \in X_{\xi}^{\prime}$.

(ii) $x^{\prime} \circ T_{m} \in L^{\infty}(\mu)_{n}^{\sim}$ for each $x^{\prime} \in X_{\xi}^{\prime}$.

(iii) $T_{m}$ is $\left(\tau\left(L^{\infty}(\mu), L^{1}(\mu)\right), \xi\right)$-continuous.

(iv) $T_{m}$ is $\sigma$-smooth.

(v) $T_{m}$ is absolutely continuous.

(vi) $m$ is $\mu$-absolutely continuous.

Proof (i) $\Longrightarrow$ (ii) Let $x^{\prime} \in X_{\xi}^{\prime}$ and $x^{\prime} \circ m \in \mathrm{ca}_{\mu}(\Sigma)$. Then by the Radon-Nikodym theorem there exists $v_{x^{\prime}} \in L^{1}(\mu)$ such that $\left(x^{\prime} \circ m\right)(A)=\int_{A} v_{x^{\prime}} d \mu$ for all $A \in \Sigma$. It follows that

$$
\left(x^{\prime} \circ T_{m}\right)(u)=\int_{\Omega} u d\left(x^{\prime} \circ m\right)=\int_{\Omega} u v_{x^{\prime}} d \mu \text { for all } u \in L^{\infty}(\mu),
$$

and this means that $x^{\prime} \circ T_{m} \in L^{\infty}(\mu)_{n}^{\sim}$. 
(ii) $\Longleftrightarrow$ (iii) $\Longleftrightarrow$ (iv) $\Longleftrightarrow$ (v) See Proposition 2.1.

(v) $\Longrightarrow$ (vi) Assume that $T_{m}$ is absolutely continuous, and let $\mu\left(A_{n}\right) \rightarrow 0,\left(A_{n}\right) \subset \Sigma$. Then $m\left(A_{n}\right)=T_{m}\left(\mathbb{1}_{A_{n}}\right) \rightarrow 0$ for $\xi$, as desired.

(vi) $\Longrightarrow$ (i) It is obvious.

As a consequence of Proposition 3.1 we get the following Pettis type theorem for countably additive measures (see [5, Theorem 1, p. 10]).

Corollary 3.2 Assume that $(X, \xi)$ is a quasicomplete lcHs. Let $m: \Sigma \rightarrow X$ be a $\xi$-countably additive measure. Then the following statements are equivalent:

(i) $m \ll \mu$.

(ii) $m$ is $\mu$-absolutely continuous.

Let $c a(\Sigma, X)$ stand for the space of all $\xi$-countably additive measures $m: \Sigma \rightarrow X$. By $c a_{\mu}(\Sigma, X)$ we denote the subspace of $c a(\Sigma, X)$ consisting of all $m \in c a(\Sigma, X)$ that are $\mu$-absolutely continuous. Denote by $\mathcal{T}_{s}$ the topology of simple convergence in $c a(\Sigma, X)$. Then $\mathcal{T}_{s}$ is generated by the family $\left\{q_{p, A}: p \in \mathcal{P}_{\xi}, A \in \Sigma\right\}$ of seminorms, where

$$
q_{p, A}(m):=p(m(A)) \quad \text { for all } \quad m \in c a(\Sigma, X) .
$$

Proposition $3.3 c a_{\mu}(\Sigma, X)$ is a closed set in $\left(c a(\Sigma, X), \mathcal{T}_{S}\right)$.

Proof Let $m \in c a(\Sigma, X)$ and $m \in \operatorname{cl}_{\mathcal{T}_{s}}\left(c a_{\mu}(\Sigma, X)\right)$. Then there is a net $\left(m_{\alpha}\right)$ in $c a_{\mu}(\Sigma, X)$ such that $m_{\alpha} \rightarrow m$ for $\mathcal{T}_{s}$, i.e., for each $p \in \mathcal{P}_{\xi}$ and $A \in \Sigma$ we have $q_{p, A}\left(m-m_{\alpha}\right)=p\left(m(A)-m_{\alpha}(A)\right) \underset{\alpha}{\longrightarrow} 0$. Assume that $\mu(A)=0$. Then $m_{\alpha}(A)=0$ for all $\alpha$, and it follows that $p(m(A))=0$ for each $p \in \mathcal{P}_{\xi}$, i.e., $m(A)=0$. In view of Corollary $3.2 m \in c a_{\mu}(\Sigma, X)$.

Now we establish some terminology (see [14, pp. 92-93]). For $p \in \mathcal{P}_{\xi}$ let $X_{p}=$ $(X, p)$ be the associated seminormed space. Denote by $\left(\widetilde{X}_{p},\|\cdot\|_{p}^{\sim}\right)$ the completion of the quotient normed space $X / p^{-1}(0)$. Let $\Pi_{p}: X_{p} \rightarrow X / p^{-1}(0) \subset \widetilde{X}_{p}$ be the canonical quotient map.

Given a vector measure $m: \Sigma \rightarrow X$ with $m \ll \mu$, let $m_{p}: \Sigma \rightarrow \widetilde{X}_{p}$ be given by

$$
m_{p}(A):=\left(\Pi_{p} \circ m\right)(A) \text { for } A \in \Sigma .
$$

Then $m_{p}$ is a Banach space-valued measure on $\Sigma$. We define the $p$-variation $\|m\|_{p}$ of $m$ by

$$
\|m\|_{p}(A):=\left\|m_{p}\right\|(A) \text { for } A \in \Sigma,
$$

where $\left\|m_{p}\right\|$ denotes the semivariation of $m_{p}: \Sigma \rightarrow \tilde{X}_{p}$. Note that $m$ is $\xi$-bounded if and only if $\|m\|_{p}(\Omega)<\infty$ for each $\xi$-continuous seminorm $p$ on $X$. Moreover, we have (see [14, Lemma 7]):

$$
\|m\|_{p}(\Omega)=\left\|T_{m}\right\|_{p}:=\sup \left\{p\left(\int_{\Omega} u d m\right): u \in L^{\infty}(\mu),\|u\|_{\infty} \leq 1\right\} .
$$


For a subset $\mathcal{M}$ of $c a_{\mu}(\Sigma, X)$ let

$$
\mathcal{K}_{\mathcal{M}}=\left\{T_{m} \in \mathcal{L}_{\tau, \xi}\left(L^{\infty}(\mu), X\right): m \in \mathcal{M}\right\}
$$

Now we are ready to state a characterization of relative compactness in the space $\left(c a_{\mu}(\Sigma, X), \mathcal{T}_{s}\right)$ in terms of the topological properties of the set $\mathcal{K}_{\mathcal{M}}$ (see [8, Theorem 7], [15, Theorem 8], [16, Theorem 2.1]).

Theorem 3.4 Let $(X, \xi)$ be a quasicomplete lcHs. Then for a set $\mathcal{M}$ in $c a_{\mu}(\Sigma, X)$ the following statements are equivalent:

(i) $\mathcal{K}_{\mathcal{M}}$ is a relatively compact set in $\left(\mathcal{L}_{\tau, \xi}\left(L^{\infty}(\mu), X\right), \mathcal{T}_{s}\right)$.

(ii) $\mathcal{K}_{\mathcal{M}}$ is $\left(\tau\left(L^{\infty}(\mu), L^{1}(\mu)\right)\right.$, $\left.\xi\right)$-equicontinuous and for each $u \in L^{\infty}(\mu)$, the set $\left\{T_{m}(u): m \in \mathcal{M}\right\}$ is relatively $\xi$-compact in $X$.

(iii) $\mathcal{M}$ is uniformly $\mu$-absolutely continuous and for each $A \in \Sigma$, the $\operatorname{set}\{m(A)$ : $m \in \mathcal{M}$ \} is relatively $\xi$-compact in $X$.

(iv) $\mathcal{M}$ is a relatively compact set in $\left(c a_{\mu}(\Sigma, X), \mathcal{I}_{s}\right)$.

Proof (i) $\Longleftrightarrow$ (ii) See Theorem 2.5.

(ii) $\Longrightarrow$ (iii) Assume that (ii) holds and let $\mu\left(A_{n} \rightarrow 0,\left(A_{n}\right) \subset \Sigma\right.$. Then using Proposition 3.1 and Corollary 2.6 for each $p \in \mathcal{P}_{\xi}$ we have

$$
\sup _{m \in \mathcal{M}} p\left(m\left(A_{n}\right)\right)=\sup _{m \in \mathcal{M}} p\left(T_{m}\left(\mathbb{1}_{A_{n}}\right)\right) \underset{n}{\longrightarrow} 0 .
$$

This means that the family $\mathcal{M}$ is uniformly $\mu$-absolutely continuous.

(iii) $\Longrightarrow$ (iv) Assume that (iii) holds. Then $\mathcal{M} \subset c a_{\mu}(\Sigma, X) \subset c a(\Sigma, X)$ and $\mathcal{M}$ is a uniformly $\xi$-countably additive set in $c a(\Sigma, X)$. Hence by [8, Theorem 7] $\mathcal{M}$ is a relatively compact set in $\left(c a(\Sigma, X), \mathcal{T}_{s}\right)$. Since $c a_{\mu}(\Sigma, X)$ is closed in $\left(c a(\Sigma, X), \mathcal{T}_{s}\right)$, we obtain that $\mathcal{M}$ is a relatively compact set in $\left(c a_{\mu}(\Sigma, X), \mathcal{T}_{s}\right)$.

(iv) $\Longrightarrow$ (i) Assume that $\mathcal{M}$ is a relatively compact set in $\left(c a_{\mu}(\Sigma, X), \mathcal{T}_{s}\right)$, and let $\left(T_{m_{\alpha}}\right)$ be a net in $\mathcal{K}_{\mathcal{M}}$. Without loss of generality, we can assume that $m_{\alpha} \rightarrow m$ for $\mathcal{T}_{s}$, where $m \in c a_{\mu}(\Sigma, X)$. We shall show that $T_{m_{\alpha}} \rightarrow T_{m}$ in $\left(\mathcal{L}_{\tau, \xi}\left(L^{\infty}(\mu), X\right), \mathcal{T}_{s}\right)$. Indeed, let $p \in \mathcal{P}_{\xi}$ and fix $\varepsilon>0$. Since $\mathcal{M}$ is a $\mathcal{T}_{s}$-bounded subset of $c a_{\mu}(\Sigma, X)$, for each $A \in \Sigma$ we have $\sup _{\alpha} p\left(m_{\alpha}(A)\right)=\sup _{\alpha} q_{p, A}\left(m_{\alpha}\right)<\infty$. Hence, since the mapping $\Pi_{p}: X \rightarrow \widetilde{X}_{p}$ is $\left(p,\|\cdot\|_{p}^{\sim}\right)$-continuous, we obtain that $\sup _{\alpha}\left\|\left(m_{\alpha}\right)_{p}(A)\right\|_{p}^{\sim}=\sup _{\alpha}\left\|\left(\Pi_{p} \circ m_{\alpha}\right)(A)\right\|_{p}^{\sim}<\infty$. In view of the Nikodym boundedness theorem (see [5, Theorem 1, p. 14]) and 3.1 we get

$$
c=\sup _{\alpha}\left\|T_{m_{\alpha}}\right\|_{p}=\sup _{\alpha}\left\|m_{\alpha}\right\|_{p}(\Omega)<\infty .
$$

Let $u \in L^{\infty}(\mu)$ be given and choose $s_{0} \in \mathcal{S}(\mu)$ such that $\left\|u-s_{0}\right\|_{\infty} \leq \frac{\varepsilon}{3 a}$, where $a=\max \left(c,\left\|T_{m}\right\|_{p}\right)$. Then there exists $\alpha_{0}$ such that $p\left(T_{m_{\alpha}}\left(s_{0}\right)-T_{m}\left(s_{0}\right)\right) \leq \frac{\varepsilon}{3}$ for $\alpha \geq \alpha_{0}$. Hence for $\alpha \geq \alpha_{0}$ we get

$$
\begin{aligned}
& p\left(T_{m_{\alpha}}(u)-T_{m}(u)\right) \\
& \quad \leq p\left(T_{m}\left(u-s_{0}\right)\right)+p\left(T_{m}\left(s_{0}\right)-T_{m_{\alpha}}\left(s_{0}\right)\right)+p\left(T_{m_{\alpha}}\left(s_{0}\right)-T_{m_{\alpha}}(u)\right)
\end{aligned}
$$




$$
\begin{aligned}
& \leq\left\|T_{m}\right\|_{p} \cdot\left\|u-s_{0}\right\|_{\infty}+p\left(T_{m}\left(s_{0}\right)-T_{m_{\alpha}}\left(s_{0}\right)\right)+\left\|T_{m_{\alpha}}\right\|_{p} \cdot\left\|s_{0}-u\right\|_{\infty} \\
& \leq a \cdot \frac{\varepsilon}{3 a}+\frac{\varepsilon}{3}+a \cdot \frac{\varepsilon}{3 a}=\varepsilon .
\end{aligned}
$$

This means that $T_{m_{\alpha}} \underset{\alpha}{\longrightarrow} T_{m}$ in $\left(\mathcal{L}_{\tau, \xi}\left(L^{\infty}(\mu), X\right), \mathcal{T}_{s}\right)$, as desired.

Recall that the general Vitali-Hahn-Saks theorem (see [7, Theorem 2.14']) says that if $\left(m_{k}\right)$ is a sequence of $\mu$-absolutely continuous measures on a $\sigma$-algebra $\Sigma$ taking values in a lcHs $(X, \xi)$, and $m(A):=\xi-\lim m_{k}(A)$ for each $A \in \Sigma$, then $m: \Sigma \rightarrow X$ is a $\mu$-absolutely continuous measure and the family $\left\{m_{k}: k \in \mathbb{N}\right\}$ is uniformly $\mu$-absolutely continuous.

Now we shall state a generalized Vitali-Hahn-Saks theorem for operators from $L^{\infty}(\mu)$ to a quasicomplete $\mathrm{lcHs}(X, \xi)$.

Theorem 3.5 Assume that $(X, \xi)$ is a quasicomplete lcHs. Let $m_{k}: \Sigma \rightarrow X$ be $\mu$ absolutely continuous measures for $k \in \mathbb{N}$ and assume that $m(A):=\xi-\lim m_{k}(A)$ exists for each $A \in \Sigma$. Then the following statements hold:

(i) $m: \Sigma \rightarrow X$ is a $\mu$-absolutely continuous measure, and the integration operator $T_{m}: L^{\infty}(\mu) \rightarrow X$ is absolutely continuous.

(ii) $T_{m}(u)=\xi-\lim _{k} T_{m_{k}}(u)$ for all $u \in L^{\infty}(\mu)$.

(iii) The family $\left\{T_{m_{k}}: k \in \mathbb{N}\right\}$ is $\left(\tau\left(L^{\infty}(\mu), L^{1}(\mu)\right)\right.$, $\left.\xi\right)$-equicontinuous.

(iv) The family $\left\{T_{m_{k}}: k \in \mathbb{N}\right\}$ is uniformly absolutely continuous.

Proof In view of the general Vitali-Hahn-Saks theorem (see [7, Theorem 2.14']) $m: \Sigma \rightarrow X$ is $\mu$-absolutely continuous, and by Proposition 3.1 $T_{m}: L^{\infty} \rightarrow X$ is absolutely continuous.

Let $p \in \mathcal{P}_{\xi}$ and fix $\varepsilon>0$. We show that $p\left(T_{m_{k}}(u)-T_{m}(u)\right) \rightarrow 0$ for each $u \in L^{\infty}(\mu)$. Indeed, since $p\left(m_{k}(A)-m(A)\right) \rightarrow 0$ for all $A \in \Sigma$, we have

$\left\|\Pi_{p}\left(m_{k}(A)-m(A)\right)\right\|_{p}^{\sim} \rightarrow 0$, i.e., $\left\|\left(m_{k}\right)_{p}(A)-m_{p}(A)\right\|_{p}^{\sim} \rightarrow 0$ for all $A \in \Sigma$.

It follows that $\sup _{k}\left\|\left(m_{k}\right)_{p}(A)\right\|_{p}^{\sim}<\infty$ for all $A \in \Sigma$, and in view of the Nikodym boundedness theorem (see [5, Theorem 1, p. 14]) and 3.1 we get

$$
a=\sup _{k}\left\|T_{m_{k}}\right\|_{p}=\sup _{k}\left\|m_{k}\right\|_{p}(\Omega)<\infty .
$$

Let $u \in L^{\infty}(\mu)$ be given and choose $s_{0} \in \mathcal{S}(\mu)$ such that $\left\|u-s_{0}\right\|_{\infty} \leq \frac{\varepsilon}{3 a}$, where $a=\max \left(c,\left\|T_{m}\right\|_{p}\right)$. Then there is $k_{0} \in \mathbb{N}$ such that $p\left(T_{m_{k}}\left(s_{0}\right)-T_{m}\left(s_{0}\right)\right) \leq \frac{\varepsilon}{3}$ for $k \geq k_{0}$. Hence for $k \geq k_{0}$ we have

$$
\begin{aligned}
p & \left(T_{m_{k}}(u)-T_{m}\left(u-s_{0}\right)\right) \\
& \leq p\left(T_{m}\left(u-s_{0}\right)\right)+p\left(T_{m}\left(s_{0}\right)-T_{m_{k}}\left(s_{0}\right)\right)+p\left(T_{m_{k}}\left(s_{0}\right)-T_{m_{k}}(u)\right) \\
& \leq\left\|T_{m}\right\|_{p} \cdot\left\|u-s_{0}\right\|_{\infty}+p\left(T_{m}\left(s_{0}\right)-T_{m_{k}}\left(s_{0}\right)\right)+\left\|T_{m_{k}}\right\|_{p} \cdot\left\|s_{0}-u\right\|_{\infty} \\
& \leq a \cdot \frac{\varepsilon}{3 a}+\frac{\varepsilon}{3}+a \cdot \frac{\varepsilon}{3 a}=\varepsilon .
\end{aligned}
$$


It follows that $T_{m_{k}} \rightarrow T$ for $\mathcal{T}_{s}$ in $\mathcal{L}_{\tau, \xi}\left(L^{\infty}(\mu), X\right)$. Since $\left\{T_{m_{k}}: k \in \mathbb{N}\right\} \cup\{T\}$ is a $\mathcal{T}_{s}$-compact subset of $\mathcal{L}_{\tau, \xi}\left(L^{\infty}(\mu), X\right)$, by Theorem 2.5 the set $\left\{T_{m_{k}}: k \in \mathbb{N}\right\}$ is $\left(\tau\left(L^{\infty}(\mu), L^{1}(\mu)\right), \xi\right)$-equicontinuous, and by Corollary 2.6 it is uniformly absolutely continuous.

Open Access This article is distributed under the terms of the Creative Commons Attribution License which permits any use, distribution, and reproduction in any medium, provided the original author(s) and the source are credited.

\section{References}

1. Aliprantis, C.D., Burkinshaw, O.: Positive Operators. Academic Press, New York (1985)

2. Aliprantis, C.D., Burkinshaw, O.: Locally Solid Riesz Spaces with Applications to Economics, 2nd edn. In: Math. Surveys and Monographs, no. 105 (2003)

3. Bourbaki, N.: Elements of Mathematics, Topological Vector Spaces, chaps. 1-5, Springer Verlag, Berlin (1987)

4. Cristescu, R.: Topological Vector Spaces. Editura Academiei, Romania (1977)

5. Diestel, J., Uhl, J.J.: Vector Measures, Amer. Math. Soc., Math. Surveys 15, Providence, RI (1977)

6. Dodds, P.G.: o-weakly compact mappings of Riesz spaces. Trans. Amer. Math. Soc. 214, 389402 (1975)

7. Drewnowski, L.: Decompositions of set functions. Studia Math. 48, $23-48$ (1973)

8. Graves, W.H., Ruess, W.: Compactness in spaces of vector-valued measures and a natural Mackey topology for spaces of bounded measurable functions. Contemp. Math. 2, 189-203 (1980)

9. Hoffman-Jorgensen, J.: Vector measures. Math. Scand. 28, 5-32 (1971)

10. Kantorovich, L.V., Akilov, A.V.: Functional Analysis. Pergamon Press, Oxford-Elmsford, New York (1982)

11. Namioka, I.: Partially ordered linear topological spaces. Mem. Amer. Math. Soc. 24 (1957)

12. Orlicz, W.: Operations and linear functionals in spaces of $\varphi$-integrable functions, Bull. Acad. Polon. Sci. Sér. Sci. Math. Astronom. Phys. 8, 563-565 (1960)

13. Orlicz, W., Wnuk, W.: Absolutely continuous and modularly continuous operators defined on spaces of measurable functions. Ricerche di Matematica 15(2), 243-258 (1991)

14. Panchapagesan, T.V.: Applications of a theorem of Grothendieck to vector measures. J. Math. Anal. Appl. 214, 89-101 (1997)

15. Schaefer, H., Zhang, X-D.: On the Vitali-Hahn-Saks theorem, Operator Theory: Advances and Applications, vol. 75, pp. 289-297. Birkhäuser, Basel (1995)

16. Zhang, X-D.: On weak compactness in spaces of measures. J. Funct. Anal. 143, 1-9 (1997) 